\title{
GASEOUS RESPONSE TO BAR-LIKE DISTORTIONS
}

William W. Roberts, Jr.

University of Virginia

In this review the large-scale dynamics of the gas in a model disk galaxy which has an oval, bar-like distortion (or bar structure) in the inner parts is reviewed from the standpoint of recent gas dynamical studies, both steady state and time evolutionary.

\section{GALACTIC SHOCKS AND LARGE NONCIRCULAR MUTIONS}

In the early 1960s Prendergast already suspected that the narrow dark dust lanes observed along the bar structure in barred spirals, such as NGC 1300 shown in Figure 1, may be related to galactic shocks formed within the gas (Prendergast, 1962). This suspicion together with the early work of Lin and Shu $(1964,1966)$ on the density wave picture of spiral structure motivated the steady state gas dynamical studies of the late 1960s (Fujimoto, 1968; Roberts, 1969; Roberts and Yuan, 1970) in which the gaseous response to a spiral perturbation gravitational field underlying a spiral density wave pattern was found to be strong and capable of inducing the formation of large-scale shock waves along the spiral arms. These studies identified the dark dust lanes along spiral arms as possible tracers of shocks and the galactic shock itself as a possible triggering mechanism for the formation of young stellar associations and $H$ II regions "strung out like pearls along the arms" (Baade, 1963).

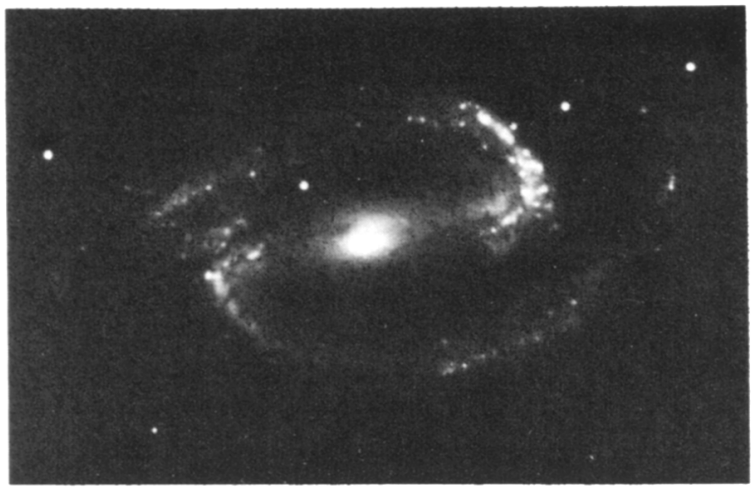

Figure 1. Photograph of the barred spiral galaxy NGC 1300 (The Hale Observatories).

W. B. Burton (ed.), The Large-Scale Characteristics of the Galaxy, 175-186. Copyright $\odot 1979$ by the IAU. 
The steady state response of the gas to a mild bar-like distortion rotating at a small angular speed $\Omega_{\mathrm{p}}$ was considered by Roberts (1971). Figure 2 shows the equipotential contours of the nonaxisymmetric gravitational field. The remarkably strong gaseous response driven by this weakly perturbed field is shown in Figure 3. Galactic shocks form in lanes slightly offset along the major axis of the bar-like distortion. These offset shocks together with the highly oval gas streamlines, large noncircular motions, and strong radial gas streaming along the bar, all driven in the presence of only weak forcing, provided a nice preview as early as 1971 of what was to come in later work.
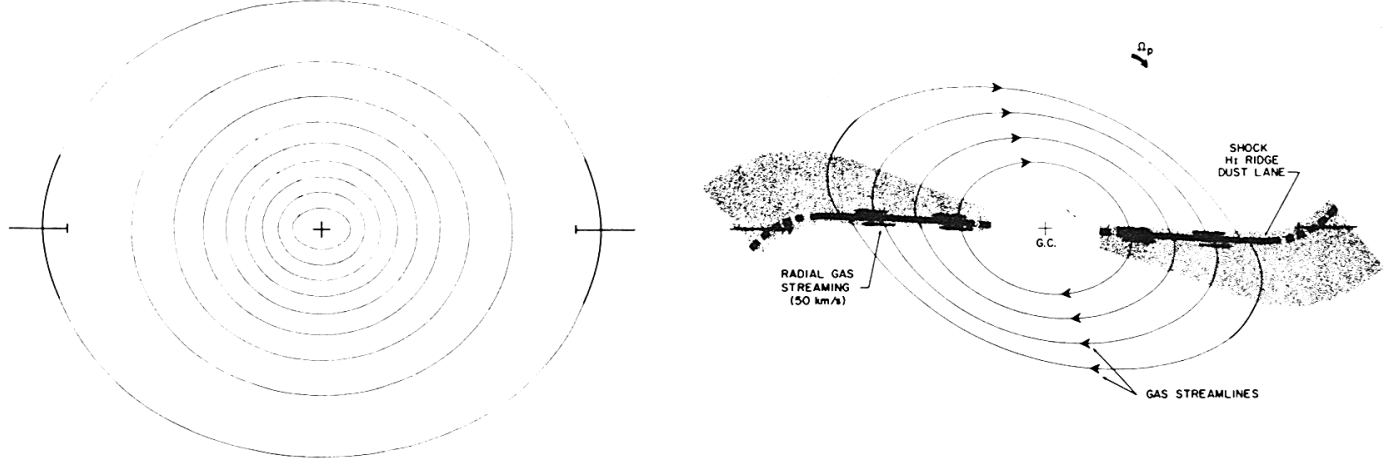

Figure 2. Equipotential contours in a model disk galaxy with a mild (10\%) bar-like distortion (Roberts, 1971).
Figure 3. Oval steady state gas circulation driven by the gravitational field of the bar-like distortion in the model disk galaxy (Fig. 2). Galactic shocks and large noncircular motions characterize the flow (Roberts, 1971).

THE "3 KPC ARM" FEATURE

Evidence for the existence of a weak bar structure in the inner parts of our Galaxy has been discussed by de Vaucouleurs $(1964,1970)$ and by Kerr (1967). Figure 4 shows the observed 21-cm velocity longitude map as derived by Peters (1975) from Kerr's (1969) 21-cm survey, galactic equator scan. The dark curve labelled (1) traces the " $3 \mathrm{kpc}$ arm" feature. The first inkling that the " $3 \mathrm{kpc}$ arm" feature could result from the gaseous response to a bar-like distortion comes from Peters (Peters and Roberts, 1972) who takes the dynamical model of Roberts (1971, see Figure 3) without any changes and views it at various orientations with respect to the galactocentric line. The $21-\mathrm{cm}$ line profiles which would be observed in our Galaxy from neutral hydrogen having the velocity and density distributions predicted in the model are determined over the longitude range: $-20^{\circ}$ to $20^{\circ}$. The results give a " $3 \mathrm{kpc}$ arm" feature for an orientation of the oval streamlines at about $135^{\circ}$ measured counterclockwise from the galactocentric line. What is remarkable: the comparison with the observed "3 kpc arm" feature shows surprisingly good agreement in view of the fact that the orientation is the only parameter adjusted. What is striking: this "3 kpc arm" feature is produced not from gas of high density, 
as in a real spiral arm, but rather from less dense gas integrated over large distances along the line of sight over the oval annular region along and between streamlines. Figure 5 shows the predicted $21-\mathrm{cm}$ velocity longitude map derived in the subsequent work of Peters (1975) in which he relaxes his dependence on the dynamical model in an attempt to account for additional features, to the extent of kinematically modelling with smoothed ellipses and allowing freedom in the choice of two parameters - the axial ratio and the mean angular momentum/unit mass - as functions of radius. Other attempts to account for the " $3 \mathrm{kpc}$ arm" feature are made by Simonson and Mader $(1972,1973)$ with an elliptical dispersion ring model and by Sanders and Prendergast (1974) with a nuclear explosion model.

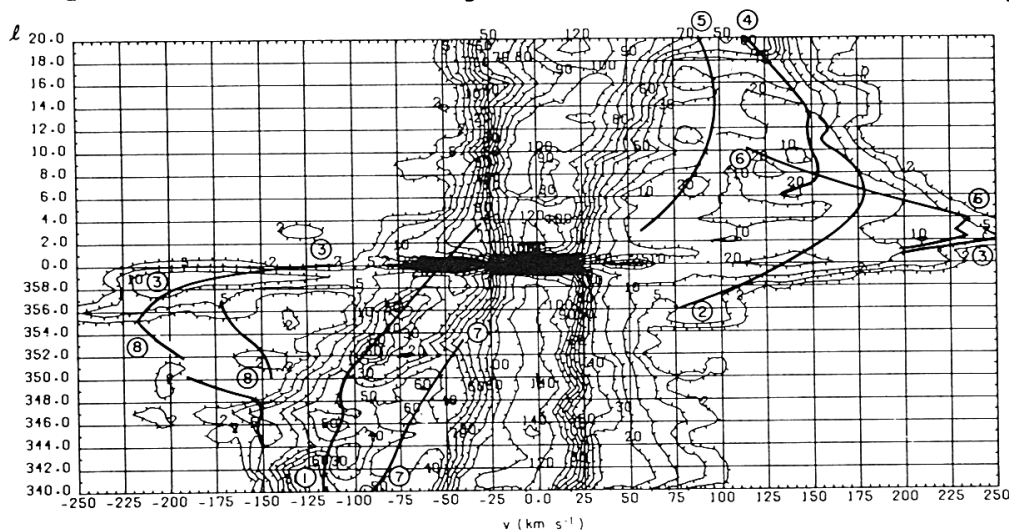

Figure 4.

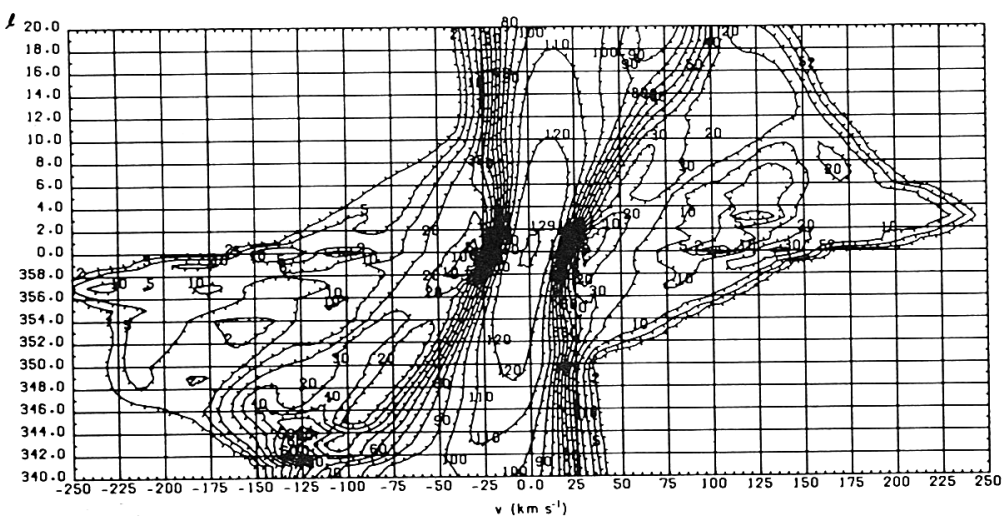

Figure 5.

Figure 4. Observed 21-cm velocity longitude map derived from Kerr's (1969) 21-cm survey, galactic equator scan. The solid curves, also from Westerhout's (1969) survey. Curve (1) - "3 kpc arm" feature (Peters, 1975). Figure 5. Predicted $21-\mathrm{cm}$ velocity longitude map derived from a kinematic elliptical flow model not greatly different from the dynamical, oval circulation model driven by a bar-like distortion (Figure 3). (Peters, 1975)

\section{STEADY STATE GAS FLOW}

Roberts and Huntley (1978; also see Roberts, Huntley, and Lin, 1977) generalize the steady state gas dynamical studies of the late 1960s for 
tightly-wound normal spirals to include normal spirals with open spiral arms and barred spirals with bar structures in the inner parts. The response of the gas to a perturbing potential that is bar-like in the interior and spiral-like in the exterior is calculated by means of an analysis which enables the two-dimensional flow to be broken up into two physical regimes, illustrated in Figure 6 . In regime I near and within the bar (and spiral arms), the flow is determined through an asymptotic approximation that neglects the small variation of the velocity, density, and pressure along a shock with respect to that variation normal to the shock. In regime II where the flow is highly supersonic, the flow is determined through an asymptotic approximation that neglects secondary terms proportional to the square of the dispersion speed, such as the transverse gradient of pressure. The composite picture for the steady state flow of gas is constructed by joining the two regimes of flow in the transition layer between regimes.
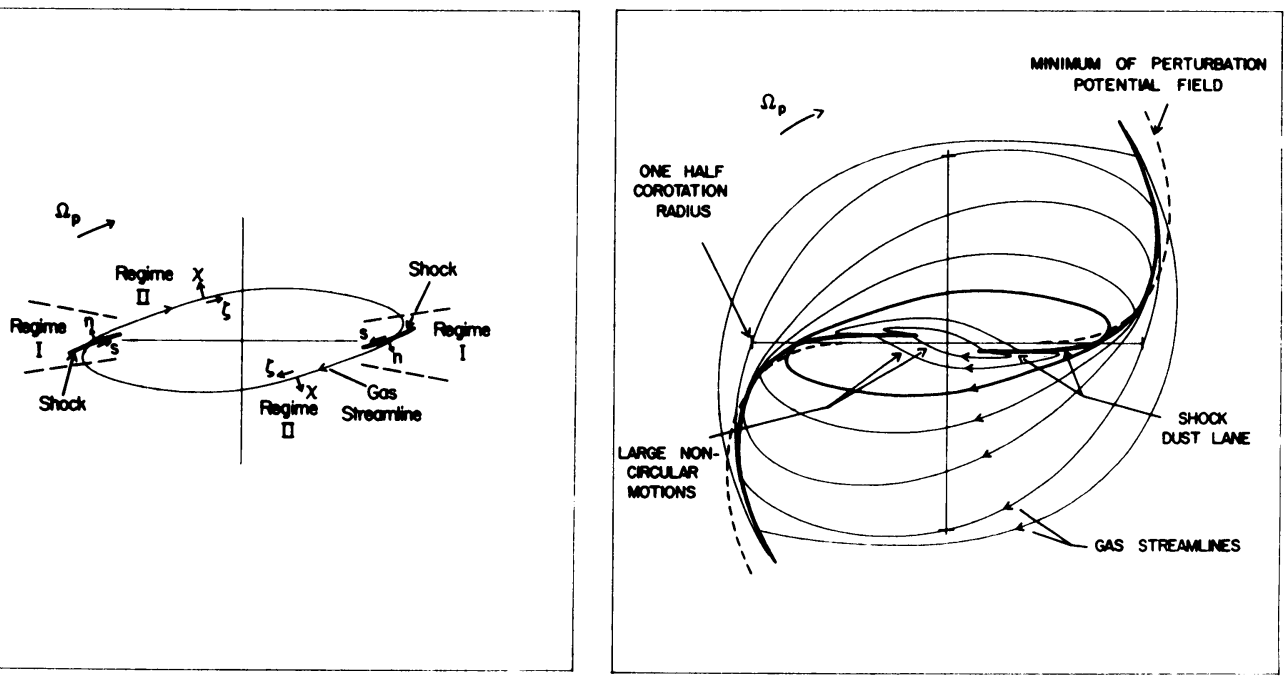

Figure 6. Schematic of the steady state flow along a streamline, and the locations of regimes I and II (Roberts and Huntley, 1978).
Figure 7. Oval steady state gas circulation in a Toomre model with a spheroidal component, driven by a mild bar-like distortion. In the inner parts the response shows offset shocks, large noncircular motions $(50-100 \mathrm{~km} / \mathrm{s})$, and radial gas streaming (Roberts and Huntley, 1978).

This composite picture is shown in Figure 7 for the case of a thin disk Toomre (1963) model with a spheroidal component superposed in the inner parts and a perturbing potential representing a $6 \%$ perturbation. Galactic shocks are present, and highly oval gas streamlines characterize the circulation in the inner parts where large noncircular motions occur. strong velocity gradients are present across the major axis of the gas bar near the shock. Because gas, in passage through an oblique shock, must leave the shock at a more oblique angle than that angle at which it entered, postshock gas in the bar region in the inner parts is directed outwards whereas postshock gas in the spiral arm region in the outer parts 
is directed inwards. This shock-focusing phenomenon, which focuses gas in the inner parts outwards and gas in the outer parts inwards, leads to enhanced concentrations of compressed gas in the region of convergence where the spiral arm bends from the bar and may account in part for the enhanced star formation activity observed at the ends of the bar structure in many barred spirals.

Figure 8 shows why shocks form. The cusp-transition curve, for any one of the four models shown, represents the critical level of forcing, at any radius, for which the gas flow undergoes a transition from flow without shocks to flow with shocks. The transition curve delineated with solid round dots (-) represents the case of the Toomre model with spheroidal component shown in Figure 7. The strong gaseous response and the formation of shocks in this model result from the moderatelystrong (6\%) forcing amplitude, here indicated by the horizontal dashed line at $A=1.2$, at a level above the transition curve.

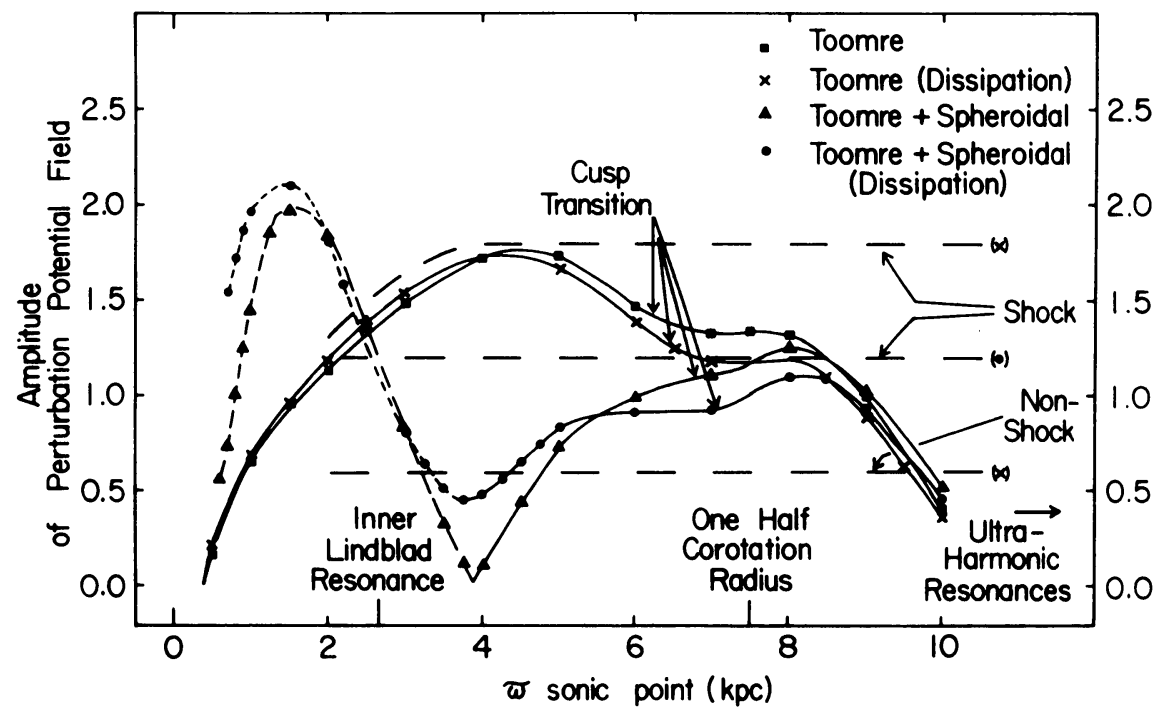

Figure 8. Amplitude of the perturbation potential field of the bar-like distortion which, at each radius, characterizes the transition from steady state flow without shocks to steady state flow with shocks. A $=2.0$ represents a $10 \%$ perturbation (at $\widetilde{w}=5 \mathrm{kpc}$ ). (Roberts and Huntley, 1978)

\section{TIME EVOLUTIONARY GAS FLOW}

Time dependent, two-dimensional, numerical hydrodynamical calculations are carried out by Sanders and Huntley (1976), Sorensen, Matsuda, and Fujimoto (1976), Huntley, Sanders, and Roberts (1978), and Liebovitch (1978) for the time evolutionary response of gas to bar-like perturbations. Sanders and Huntley for a power law disk model with a mild oval distortion and Sorensen, Matsuda, and Fujimoto for an Eggen, Lynden-Bell, and Sandage disk model with a strong prolate spheroidal distortion both found in 1976 the result that the time evolutionary gas response shows open trailing 

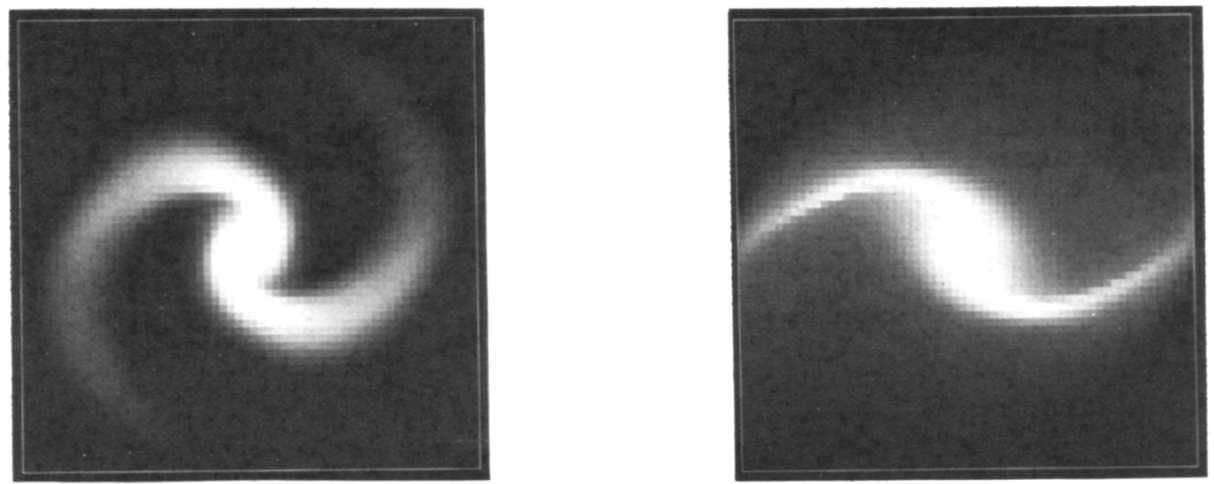

Figure 9. Photographic simulation (a snapshot) of the time evolutionary gas response in a power law disk model to a mild bar-like distortion for two cases: left - inner and outer Lindblad resonance and corotation are contained within the gaseous disk; right - only inner Lindblad resonance is contained within the gaseous disk (Sanders and Huntley, 1976, as recalculated in the inertial frame by Huntley, 1977).

spiral density waves rotating with the angular speed of the distortion. Figure 9 from Sanders and Huntley shows a photographic simulation of the gas density distribution for the power law disk model as a snapshot in time for two such cases. The shape of the spirals can be interpreted through the coherent shifting in orientation of periodic particle orbits between and across the resonances and corotation.

Figure 10 from Huntley, Sanders, and Roberts for a Toomre model with a mild bar-like distortion shows a gas response in the form of a prominent central gas bar, with open trailing gaseous spiral density waves. Sanders (1977) finds that the response of an ensemble of non-interacting test particles to a bar-like distortion is bar-like, without appreciable spiral arm formation; whereas when he repeats the calculation in a way in which the test particles take on the character of a gas with an artificial viscosity, he finds the response to be bar-like inside inner Lindblad resonance, with two trailing spiral density waves outside. This result indicates that the gaseous spiral density waves form through the dissipation provided by the artificial viscosity in the numerical codes. Viscous effects also induce a strong perennial inward drift of gas toward the center.

Sorensen, Matsuda, and Fujimoto in their model with strong prolate spheroidal distortion find the time evolutionary response to be offset toward the leading edges of the bar. With higher resolution, Huntley, Sanders, and Roberts consider a similar case of a Toomre model with a prolate spheroidal distortion, and a photographic simulation of this is shown in Figure 11. The gas response in the inner parts is quite strong and shows distinctly-offset lanes along the leading edges of the underlying bar-like prolate spheroid. Of course there is still a problem of resolution, directly related to the smoothing effects of artificial viscosity in the numerical code; nevertheless, it is tempting to identify these offset features as tracers of large-scale shocks in the gas. 


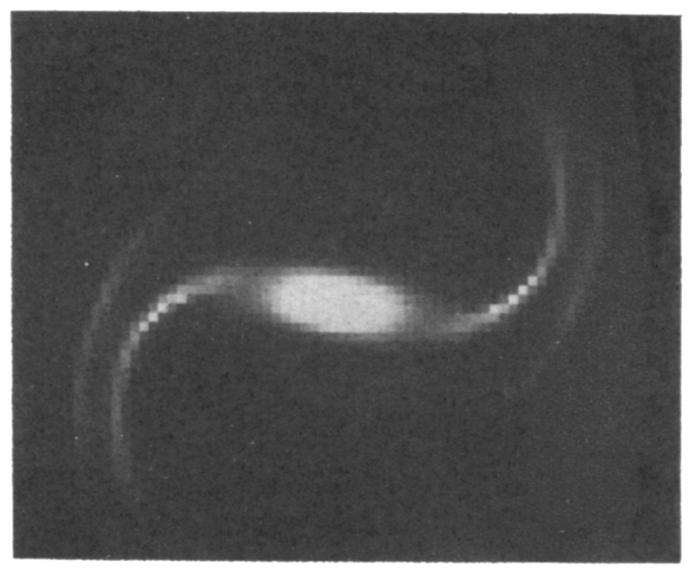

Figure 10. Photographic simulation (a snapshot) of the time evolutionary gas response in a Toomre model to a mild bar-like distortion (Huntley, Sanders, and Roberts, 1978).

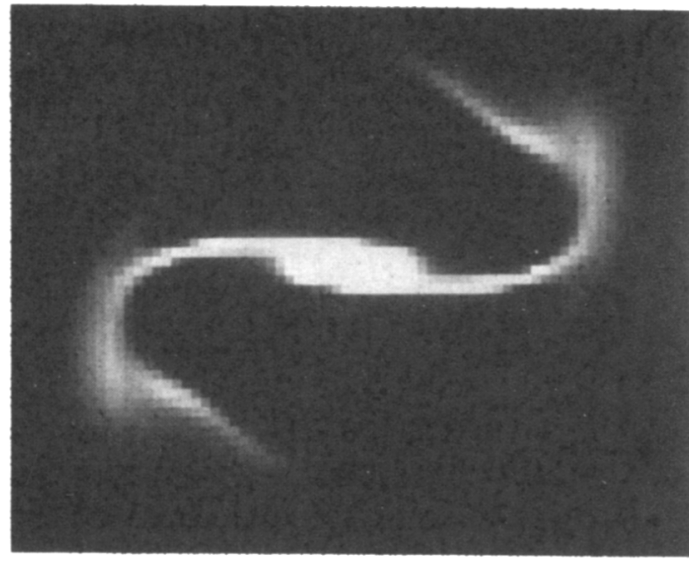

Figure 11. Photographic simulation (a snapshot) of the time evolutionary gas response in a Toomre model to a prolate spheroidal distortion (Huntley, Sanders, and Roberts, 1978).

In order to focus in more detail on these features, we will return to the steady state model (Figure 7) where viscous smoothing does not enter. Figure 12 shows the velocity field for this steady state model in a display which plots velocity vectors at approximately $6400 \mathrm{cell}$ positions over an 80 by 80 grid, 1 cell/300 pcs here, which is the identical grid size used in the time evolutionary calculations. Numerous arrows delineate the strong outward preshock flow approaching the shock and also the strong inward postshock flow at distances downstream from the shock. Only two arrows are apparent which delineate the outward, highly oblique postshock flow just adjacent to the shock. Corresponding velocity field displays for the time evolutionary calculations show a strikingly similar character; except in the narrow postshock region just adjacent to the shock, not even one arrow can be found which delineates outward, highly oblique postshock flow. The artificial viscosity is no doubt playing a role in the time evolutionary calculations to smooth such fine features. On the other hand the resolution of even the best, current observations is coarser; and to that degree of resolution, such differences in the theoretical displays would not even be detectable.

Recent time evolutionary, two dimensional, numerical calculations by Liebovitch (1978) on the gas response to an imposed perturbing potential that is bar-like in the interior and spiral-like in the exterior, on a polar coordinate grid, provide important confirmations of many of the results found in these earlier studies. Liebovitch confirms such effects as the offsetness of the gas features along the bar. Figure 13 shows his determination of the clockwise phase shift toward $90^{\circ}$ of the gas bar relative to the (horizontal) driving bar, for the case of a disk model with one inner Lindblad resonance when the amplitude of the driving bar is 


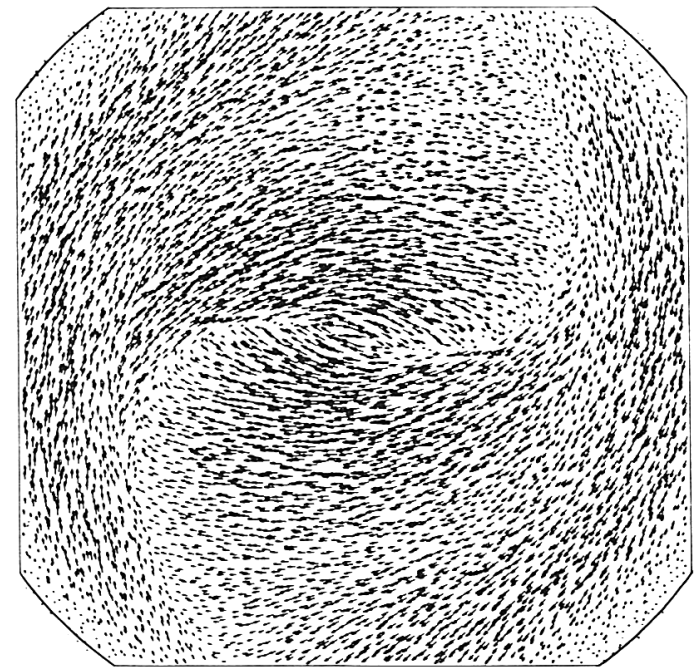

Figure 12. Velocity field of the steady state model in Figure 7 , delineated by some 6400 velocity vectors. To this degree of resolution, shocks are detectable (Roberts and Huntley, 1978).

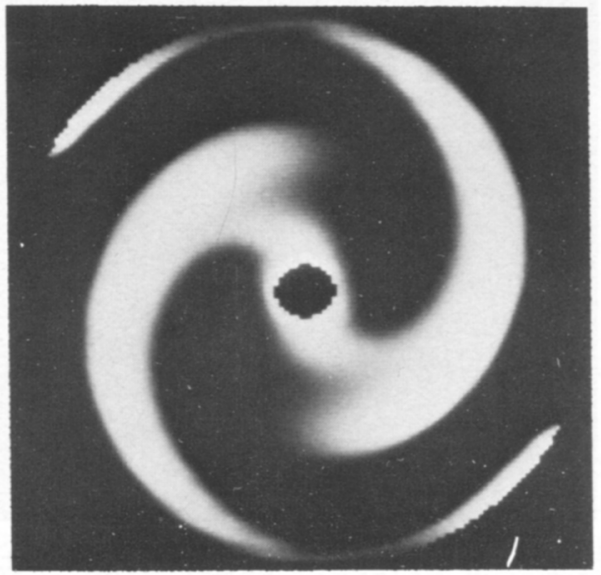

Figure 13. Photographic simulation of the gas density distribution for a case in which the gas bar response shifts to approximately $90^{\circ}$ out of phase with the driving bar (Liebovitch, 1978).

reduced toward zero. In addition, he finds broad appendages of high gas density that extend past the bend where the central bar bends into spiral arms (most likely related to the shock-focusing phenomenon discussed earlier); and these he associates with the bright appendages to the spiral arms seen in many barred spirals.

\section{A SAMPLE BARRED SPIRAL}

One of the most detailed velocity field maps for barred spirals available to date in the literature is that of NGC 5383 (photograph shown in Figure 14) derived from spectroscopic observations by Peterson,

Figure 14. Photograph of the barred spiral galaxy NGC 5383 (The Kitt Peak National Observatory).

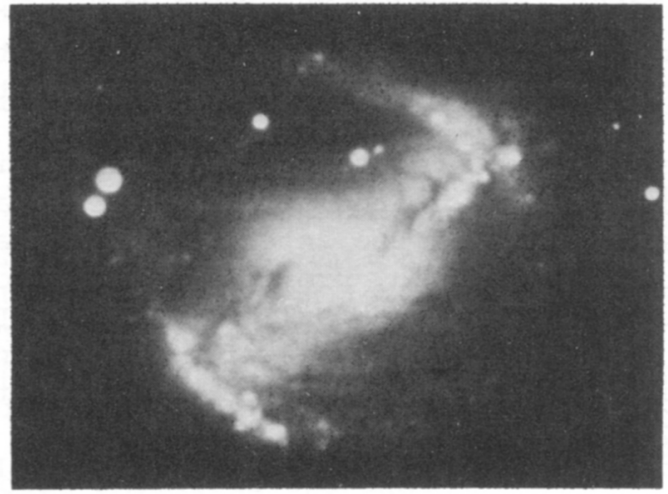


Rubin, Ford, and Thonnard (1978). Figure 15 shows this observed velocity field map. Probably what is most striking: the strong velocity gradient across the bar, as evidenced by the crowding of isovelocity contours there Such a strong velocity gradient may be directly related to the bar-driven flow of gas in NGC 5383 .

In order to investigate this possibility we will adopt the steady state model (Figures 7 and 12) and view it in the same orientation as NGC 5383 is viewed in the sky. Figure 16 from Roberts and Huntley (1978) shows the velocity field map (solid lines) predicted from the steady state model; independently Huntley (1978) has followed a similar procedure for a time evolutionary model. As in the observed map, what is most striking in Figure 16 is the strong gradient of velocity across the bar, which in the model is a result of the highly oval gas circulation driven by the bar-like potential field. Overall there seems to be general agreement between these isovelocity contours and those in the observed map. Perhaps of further interest is the trough in the -50 and $-100 \mathrm{~km} / \mathrm{s}$ isovelocity contours in the NW quadrant where these contours cross the shock and spiral arm, and also the corresponding trough in the 50 and $100 \mathrm{~km} / \mathrm{s}$ isovelocity contours in the SE quadrant. Similar troughs show up in the observed map in the -100 and $100 \mathrm{~km} / \mathrm{s}$ isovelocity contours in the regions where these contours cross the arms near the ends of the bar. If further observations of this type could be made, particularly near and along the bar structure and spiral arms, corresponding troughs and other detailed variations in more of the observed isovelocity contours might be detectable and help to further reflect the underlying dynamics.

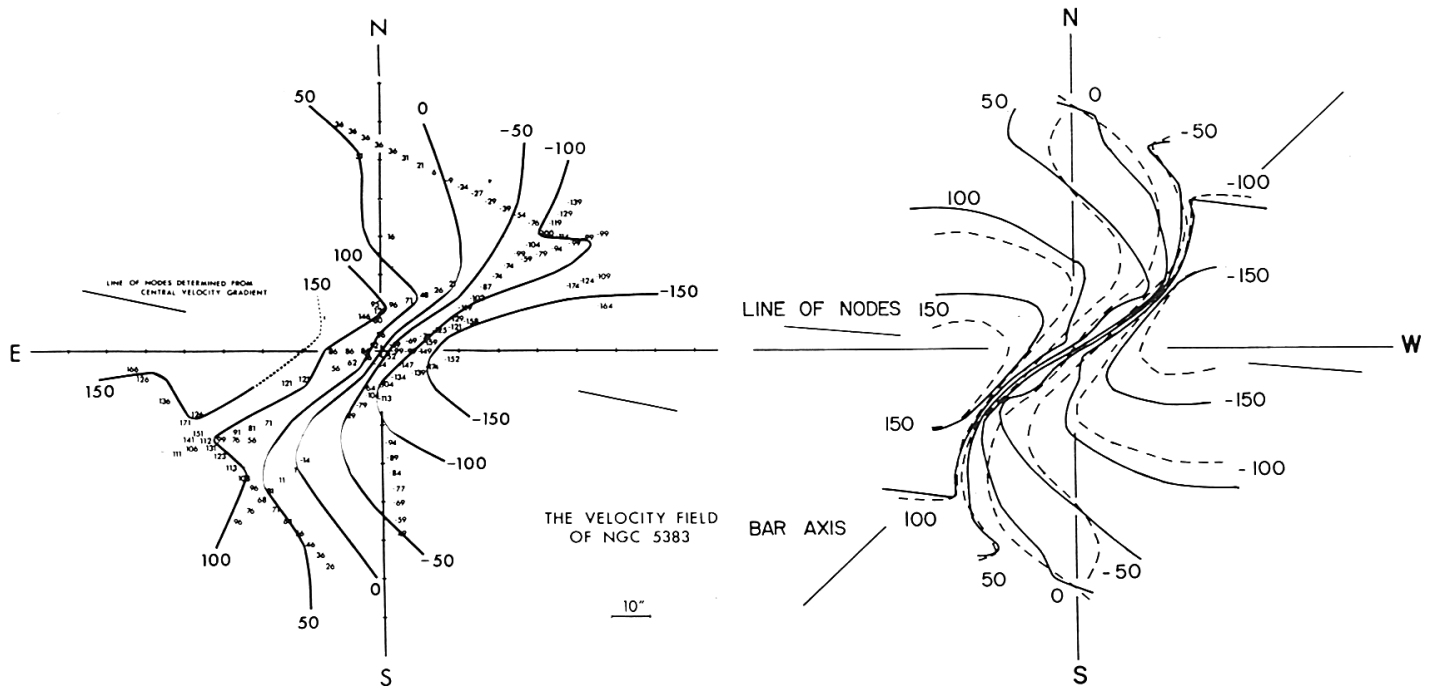

Figure 15. Observed velocity field of the barred spiral galaxy NGC 5383 characterized by a strong gradient in velocity across the bar (Peterson, Rubin, Ford, and Thonnard, 1978).
Figure 16. Predicted velocity field of the steady state model in Figures 7 and 12, with the same orientation as that observationally-derived for NGC 5383 (Roberts and Huntley, 1978). 
OUR GALAXY - NOT A BIRD'S EYE VIEW

Does our Galaxy contain a bar-like distortion or bar structure in the inner parts? If so, how prominent is it and what characteristics does it share in common with the bar structures in NGC 1300 or NGC 5383 or other external barred spirals which we see with a bird's eye view? Perhaps the results and implications of the studies reviewed herein will motivate future studies, both observational and theoretical, to help us focus further on this intriguing question, toward a deeper understanding of our Galaxy.

I would like to thank Jim Huntley, Larry Liebovitch, Bill Peters, and Charles Peterson for providing photographs included in this review. Portions of this work were supported in part by the National Science Foundation under grant AST72-05124 A04.

\section{REFERENCES}

Baade, W., 1963, "Evolution of Stars and Galaxies," Harvard U., Cambridge. de Vaucouleurs, G., 1964, in IAU Symp. No. 20, p. 195.

de Vaucouleurs, G., 1970, in IAU Symp. No. 38, p. 18.

Fujimoto, M., 1968, in IAU Symp. No. 29, p. 453.

Huntley, J. M., 1977, Ph. D. Thesis, U. of Va., Charlottesville.

Huntley, J. M., 1978, preprint.

Huntley, J. M., Sanders, R. H., Roberts, W. W., 1978, Ap. J., 221, p. 521. Kerr, F. J., 1967, in IAU Symp. No. 31, p. 239.

Kerr, F. J., 1969, Australian J. Phys., Ap. Suppl., No. 9.

Liebovitch, L. S., 1978, Ph. D. Thesis, Harvard U., Cambridge.

Lin, C. C., and Shu, F. H., 1964, Ap. J., 140, p. 646.

Lin, C. C., and Shu, F. H., 1966, Proc. Natl. Acad. Sci., 55, p. 229.

Peters, W. L., 1975, Ap. J., 195, p. 617.

Peters, W. L., and Roberts, W. W., 1972, B.A.A.S., 4, p. 265.

Peterson, C. J., Rubin, V. C., Ford, W. K., Thonnard, N., 1978, Ap. J., $219, \mathrm{p} .31$.

Prendergast, K. H., 1962, in Interstellar Matter in Galaxies,

L. Woltjer (ed.), Benjamin, N. Y.

Roberts, W. W., 1969, Ap. J., 158, p. 123.

Roberts, W. W., 1971, B.A.A.S., 3, p. 369.

Roberts, W. W., and Huntley, J. M., 1978, Ap. J. (submitted for publ.).

Roberts, W. W., Huntley, J. M., Lin, C. C., 1977, in IAU Symp. No. 77.

Roberts, W. W., and Yuan, C., 1970, Ap. J., 161, p. 877.

Sanders, R. H., 1977, preprint.

Sanders, R. H., and Huntley, J. M., 1976, Ap. J., 209, p. 53.

Sanders, R. H., and Prendergast, K. H., 1974, Ap. J., 188, p. 489.

Simonson, S. C., and Mader, G. L., 1972, B.A.A.S., 4, 266.

Simonson, S. C., and Mader, G. L., 1973, Astr. and Ap., 27, p. 337.

Sorensen, S. A., Matsuda, T., and Fujimoto, M., 1976, Ap. Space Sci., 43, p. 491 .

Toomre, A., 1963, Ap. J., 138, p. 385.

Westerhout, G., 1969, Maryland-Green Bank Galactic 21-cm Line Survey, 2nd ed. 


\section{DISCUSSION}

van den Bergh: Radio observations show that there is a small area of very active star formation in the center of our Galaxy. In this respect the Galaxy resembles external galaxies with nuclear hot spots. About two-thirds of the objects with such hot spots are barred spirals. This suggests that there is perhaps a $60 \%$ to $70 \%$ chance that the Galaxy also contains a nuclear bar.

Contopoulos: I noticed (in one of your slides) that the streamlines inside the bar are very elongated. According to my calculations this should happen mainly near the Inner Lindblad Resonance. Where is the Inner Lindblad Resonance in your model?

Roberts: In the steady-state model shown in Figure 7 of my paper, highly oval gas streamlines occur in the vicinity of the Inner Lindblad Resonance and in fact pass across this resonance region. In the (kpc) units of Figures 7 and 8 the half co-rotation radius occurs at $7.5 \mathrm{kpc}$ (marked, and just outside the bend of the bar into spiral arms); the Inner Lindblad Resonance, at $2.6 \mathrm{kpc}$; and the maximum and minimum radii of the second innermost streamline, at 4.0 and $1.0 \mathrm{kpc}$, respectively.

Sanders: It seemed to me that in the steady-state calculations made by you and Huntley the co-rotation radius was near the end of the bar; that is, the bar is a fast bar. I know that with respect to the time dependent calculations, it is certainly the case that co-rotation must lie near the end of the bar in order to get reasonable offset shocks. Now we lie in a region of spiral structure. This would suggest--if our galaxy is a barred spiral like NGC 1300--we lie beyond co-rotation. On the other hand, the fact that active star formation is occurring in the region of the Sun implies that we lie inside co-rotation. This apparent contradiction suggests to me that our Galaxy is not a barred spiral like NGC 1300 .

Roberts: I agree that a fast bar would result if co-rotation occurs as far inwards as the radius at the ends of the bar. However, the bar in NGC 1300 may not be so fast a rotator. First, the co-rotation radius in the steady-state mode1, shown in my Figure 7, actually lies at the outer edge of the disk outside the spirals; the bar is a slowly-rotating bar, and the shocks occur offset with about the same offsetness as the dark dust lanes observed along the bar in NGC 1300. Second, in the observed photograph of NGC 1300 the dust lanes on the inner edges of the spiral arms can be traced to a considerable radius; this implies that corotation occurs outside this radius. These considerations do not support your chain of arguments. I believe that the evidence available at this time does not rule out the possibility that our Galaxy may have a bar or bar-like distortion like that of NGC 1300. However, I do agree that such a bar or bar-like distortion in our Galaxy would probably be a slow rotator. 
Mark: Dr. Roberts showed that even a weak driving force in the form of a bar plus open spiral would result in strong bar-like gas shocks. A relevant question is whether or not the gas would react with strong shocks to the modes we calculated which sometimes have the appearance of an open spiral in the inner parts of the galaxy model. My guess is that the gaseous response might not look like NGC 1300 because the perturbation densities and potentials do not reach the galactic center in our modes. The situation could be different if the amount of bulge matter is somewhat less than that used in our N-body simulation with Bob Berman. In that case a weak bar or oval distortion forms in the central regions and the gaseous response might indeed behave as in $\mathrm{Dr}$. Roberts' simulation.

Sanders: Let me elaborate on the point I made a minute ago about fast bars. We have recently been investigating the gas response to bars by means of time-dependent hydrodynamical calculations. The model for the gravitational field consists of a homogeneous prolate spheroid embedded in a disk. We have been exploring the parameter space of this model and find that the gas response looks most similar to a barred spiral like NGC 1300 whenever co-rotation lies just beyond the ends of the bar. This is consistent with calculations made several years ago by Prendergast which suggest that the location of the straight dust lanes depend critically upon the presence of Lagrangian points near the ends of the bar.

van Woerden: The pile up of gas just outside a bar may be seen well in the barred Magellanic irregular NGC 4449. A Westerbork map of neutral hydrogen in this galaxy (by Bosma and myself, in preparation) shows a very strong ridge of hydrogen at the $\mathrm{NE}$ end of the bar, coinciding with a chain of bright HII regions and associations.

de Vaucouleurs: I should like to call your attention to the on-going program of Fabry-Perot interferometry at McDonald Observatory, including many barred spirals. The first results will appear in the dissertation of $W$. Pence; this is a study of the velocity field in NGC 253 from about 10,000 individual velocities.

Roberts: I look forward to the publication of this high-resolution study which perhaps can be compared with high-resolution theoretical velocity-field maps, such as the one shown in my Figure 12, to help guide future theoretical studies. 